\title{
Uso profiláctico y terapéutico de la ivermectina contra el virus SARS CoV-2
}

\author{
Prophylactic and therapeutic use of ivermectin against SARS CoV-2 virus
}

Tito Alvarado Matute. 이

Médico Maestría en Ciencias, Salud Pública y Medicina Tropical; Infectólogo Asociado al Hospital Escuela; Hospital Medical Center; Tegucigalpa.

La ivermectina es una droga antiparasitaria ampliamente utilizada por décadas en países tropicales y subtropicales para tratar enfermedades parasitarias, considerándose muy segura y efectiva, por lo que la Organización Mundial de la Salud (OMS) la tiene incluida en la lista de las "drogas esenciales". ${ }^{1}$ Además, la ivermectina ha sido considerada como una droga antiviral y anti-inflamatoria.

La Ivermectina fue descubierta por accidente y desarrollada a finales de 1970 por Satochi Omura (Instituto Kitasato de Japón) y William Campbell (Laboratorio Merck Sharp \& Dohme USA), ${ }^{2}$ al estudiar una cepa bacteriana de Streptomyces avermectinius proveniente del suelo de un campo de golf japonés de donde se aislaron las avermectinas. Estas resultaron tener una poderosa actividad antiparasitaria, siendo la ivermectina el derivado antiparasitario de más amplio espectro (helmintos y ectoparásitos). La ivermectina es una lactona macrocíclica que, además, tiene una actividad antiviral múltiple in vitro (dengue, fiebre amarilla, zika, chikungunya, VIH, fiebre del Nilo, entre otros), incluyendo al SARS-CoV-2, cuyo mecanismo de acción es la inhibición de importinas, que son proteínas que incorporan el virus al núcleo, impidiendo de esta manera, la replicación del mismo. ${ }^{3}$ La droga es liposoluble, se absorbe por vía oral con estómago lleno, se metaboliza en hígado, y se almacena en la grasa corporal. Su vida media es de 18 horas y se excreta en heces en aproximadamente 12 días. Hace más de 30 años, la compañía Farmacéutica Merck y OMS emprendieron una campaña masiva utilizando la ivermectina para erradicar, varios años más tarde, la oncocercosis, causante de la ceguera del río que sufrían millones de africanos, y por cuya actividad, estos investigadores recibieron en el año 2015, el premio Nóbel de Medicina. ${ }^{4}$ Ha tenido un amplio uso durante 40 años en el tratamiento de enfermedades parasitarias (oncocercosis, estrongiliodiasis, filariasis, etc.) en billones de personas en el mundo. ${ }^{4}$

Recibido: 17-03-2021 Aceptado: 12-05-2021 Primera vez publicado en línea: 17-05-2021 Dirigir correspondencia a: Dr. Tito Alvarado Matute

Correo electrónico: titoalvaradom@yahoo.com

Declaración de relaciones $y$ actividades financieras y no financieras y conflictos de interés: ninguno.

DOI: https://doi.org/10.5377/rmh.v89i1.11583

(C) 2021 Autor(es): (c) (i)
La rápida expansión del coronavirus motivó a un grupo de investigadores australianos, a demostrar in-vitro (células vero), que la Ivermectina, en tan solo 48 horas, inhibía la replicación del SARS CoV-2; recientemente, esta droga ha inhibido la replicación in-vivo en ratones. ${ }^{5}$ Estos hallazgos constituyen el inicio de múltiples estudios que evidencian eficacia de la ivermectina en el tratamiento temprano y profiláctico del SARS CoV-2. ${ }^{6,7}$ Las siguientes aseveraciones dan testimonio de lo expresado: 1) Tratamientos con dosis de $200 \mathrm{mcg} / \mathrm{kg}(0.2 \mathrm{mg} / \mathrm{kg})$ de peso corporal, han mostrado raros y leves efectos adversos, evidenciando ser un medicamento inocuo y seguro, ${ }^{6}$ 2) Está documentada y demostrada la inhibición del SARS CoV-2 in-vitro e in-vivo (ratones); ${ }^{5} 3$ ) Estudios en grupos de población en riesgo, demuestran una reducción significativa de la infección del virus SARS-CoV-2, tras el uso de ivermectina en dosis semanales $^{8}$ (Ver Cuadro 1); 4) Estudios en pacientes afectados por COVID-19, muestran una reducción de la mortalidad cuando se usa ivermectina en fase temprana de la enfermedad. ${ }^{6,7}$

Su uso en profilaxis y tratamiento de COVID-19, se ha extendido a más de 18 países en 5 continentes. Así lo evidencian revisiones por separado de tres recientes meta-análisis de 44 y 18 estudios (meta-análisis, estudios aleatorizados, controlados, observacionales, serie de casos, etc.). ${ }^{8,9}$ Front Line Critical Care Alliance (FLCCC) resumió los hallazgos, analizando la efectividad de ivermectina en el tratamiento y profilaxis de la enfermedad de la COVID-19 (Cuadro 1). Su conclusión fue que la ivermectina demostró una robusta actividad terapéutica contra COVID-19 y recomendaron usarla globalmente en su manejo. La dosis recomendada por el doctor Carvallo, es de $200 \mathrm{mcg} / \mathrm{kg}(0.2 \mathrm{mg} /$ $\mathrm{kg}$ ) de peso corporal, ${ }^{6}$ y en otras publicaciones se calcula por kilo/ libra de peso, ${ }^{10}$ tal como se muestra en el Cuadro 2. La ivermectina solo debe ser prescrita por un facultativo médico. Se recomienda tomar las tabletas con estómago lleno, nunca en ayunas, ya que, de esa manera, su absorción es casi completa. La cantidad de tabletas indicada para profilaxis, se administra en una sola dosis. La dosis se repite de manera semanal durante 8 a 10 semanas. La conducta a seguir después de ese período, dependerá de la situación de la COVID-19 en el país, y si la persona se aplica la vacuna contra el COVID-19, la ivermectina se debe continuar al menos 2 semanas después de que se aplique la segunda dosis. Existen algunas contraindicaciones. No se debe usar en niños con peso inferior a $15 \mathrm{~kg}$, en mujeres embarazadas, en 
Cuadro 1. Resultados de meta-análisis: eficacia ivermectina por estadio de tratamiento, 2020-2021 (adaptado de referencia 8 y sus actualizaciones).

\begin{tabular}{|c|c|c|c|c|}
\hline $\begin{array}{l}\text { Estadio de } \\
\text { Tratamiento }\end{array}$ & $\begin{array}{c}\text { Número de Estudios } \\
\text { con resultados } \\
\text { positivos }\end{array}$ & $\begin{array}{l}\text { Porcentaje de Estudios } \\
\text { con resultados positivos }\end{array}$ & $\begin{array}{l}\text { Probabilidad de que un } \\
\text { resultado positivo sea inefectivo }\end{array}$ & $\begin{array}{c}\text { Resultados de meta-análisis } \\
\text { RR (IC95\%) }\end{array}$ \\
\hline Temprano & 15 & 100.0 & $\begin{array}{l}0.000031 \\
1 / 33000\end{array}$ & $\begin{array}{c}82 \% \text { de mejoría } \\
0.18(0.12-0.17) \\
p<0.0001\end{array}$ \\
\hline Tardío & 18 & 100.0 & $\begin{array}{c}0.0000038 \\
1 / 262000\end{array}$ & $\begin{array}{c}51 \% \text { de mejoría } \\
0.49(0.37-0.46) \\
p<0.0001\end{array}$ \\
\hline Profilaxis & 11 & 100.0 & $\begin{array}{c}0.00049 \\
1 / 2000\end{array}$ & $\begin{array}{c}89 \% \text { de mejoría } \\
0.11(0.05-0.021) \\
p<0.0001\end{array}$ \\
\hline Todos los estudios & 44 & 100.0 & $\begin{array}{c}0.000000000000057 \\
1 / 18 \text { trillones }\end{array}$ & $\begin{array}{c}75 \% \text { de mejoría } \\
0.25(0.19-0.34) \\
p<0.0001\end{array}$ \\
\hline
\end{tabular}

Cuadro 2. Ivermectina: estimación de la dosis por kilogramo y por libra de peso (adaptado de referencia 10).

\begin{tabular}{|c|c|c|}
\hline \multicolumn{2}{|c|}{ Peso } & \multirow{2}{*}{$\begin{array}{c}\text { Tabletas } 6 \mathrm{mg} \\
\text { Cantidad }\end{array}$} \\
\hline $\mathrm{Kg}$ & Lb & \\
\hline $15-20$ & $33-44$ & $1 / 2$ \\
\hline $20.1-40$ & $45-88$ & 1 \\
\hline $40.1-50$ & $89-110$ & $11 \frac{2}{2}$ \\
\hline $50.1-70$ & $111-154$ & 2 \\
\hline $70.1-80$ & $155-176$ & $21 / 2$ \\
\hline $80.1-100$ & $177-220$ & 3 \\
\hline $100.1-110$ & $221-242$ & $31 / 2$ \\
\hline $110.1-+$ & $243-+$ & 4 \\
\hline
\end{tabular}

personas con trastornos hepáticos severos, en personas con antecedentes de hipersensibilidad al medicamento. ${ }^{3}$ Tampoco usar en pacientes epilépticos que están tomando barbitúricos 0 benzodiacepinas, debido a potenciales interacciones. Asimismo, no usar simultáneamente con anticoagulantes heparinas y warfarina, ya que puede interferir con el efecto de estas drogas. ${ }^{8}$

Teniendo en cuenta la tendencia creciente de los casos de COVID-19 a nivel nacional e internacional, así como la amenaza de nuevas cepas con mayor transmisibilidad y severidad, se considera probable que el riesgo de contagio entre la población y sus familias, se incremente. Creemos que es prudente que la Secretaría de Salud, además de implementar medidas de bioseguridad (mascarilla, distanciamiento físico y lavado de manos), en las personas con elevado riesgo de infección del virus SARSCoV-2 (personal de salud, personas en confinamiento, personal de seguridad, personas de la tercera edad, con o sin comorbilidades, etc.), además de incluir la ivermectina en la normativa como tratamiento MAIZ (mycrodacin, azitromicina, ivermectina, zinc) y CATRACHO (colchicina, antiinflamatorio, tocilizumab, ivermectina, anticoagulante, cloroquina, alto flujo de oxígeno, pronación), también debería recomendar su uso como profiláctico.

La decisión de recomendar el uso profiláctico de la ivermectina ha sido tomada sobre la base de investigaciones que a su vez, se fundamentan en múltiples estudios internacionales (aleatorizados, controlados, observacionales, poblacionales, serie de casos, etc.). ${ }^{6-9}$ Estos estudios han evidenciado un efec- to beneficioso de la ivermectina, vinculado a sus propiedades antivirales y antiinflamatorias, resultando en la reducción de la tasa de infección, disminución de la sintomatología y mortalidad en grupos de población que han usado esta droga en la profilaxis y/o tratamiento de la COVID-19. Hay experiencias personales que evidencian en forma aislada, la eficacia de la ivermectina como profilaxis en muchos hondureños y en grandes grupos empresariales con miles de trabajadores; así como la reducción de la mortalidad por COVID-19, después de la implementación y oficialización ministerial de los denominados protocolos de tratamiento (MAIZ y CATRACHO) que contienen dentro de su fórmula la ivermectina. ${ }^{11}$

Como sinopsis, señalamos que se reconoce que el uso de la ivermectina en el manejo del COVID-19 es un tema controversial dentro del gremio médico. No hay una aprobación oficial por algunas autoridades sanitarias nacionales e internacionales del uso profiláctico/tratamiento de la ivermectina, aduciendo cuestiones metodológicas de los estudios realizados. Sin embargo, al ponderar la amenaza de la COVID-19 frente a los escasos efectos adversos de la ivermectina, se concluye que la relación riesgo-beneficio es favorable. No obstante, información reciente indica que el Instituto Nacional de Salud de Enfermedades Infecciosas y Alergia de Estados Unidos y la OMS, están analizando que, además de su utilización en ensayos clínicos, esta droga debería ser prescrita por médicos de acuerdo a su criterio y al de sus pacientes.

En conclusión, el uso de ivermectina como tratamiento temprano, reduce la tasa de muerte del COVID-19. ${ }^{7}$ No obstante, se necesitan más estudios controlados para confirmar su eficacia, sobre todo en pacientes con enfermedad tardía. Existe evidencia científica de que el uso de ivermectina como droga profiláctica, previene la infección del SARS-CoV- $2,{ }^{6}$ sin embargo, esta debería complementarse con las medidas de bioseguridad y nunca como una estrategia aislada. La ivermectina es una droga segura, con muy pocos efectos secundarios, que siempre debe ser prescrita por un médico. Es de urgente necesidad que la Secretaría de Salud normatice el uso profiláctico de la ivermectina bajo supervisión médica, en personal con elevado riesgo de adquirir la infección por SARS-CoV-2, además, debería realizar estudios clínicos de profilaxis en el país. 


\section{REFERENCIAS}

1. World Health Organization. Model list of Essential Medicine. [Internet] Geneve, Switzerland: WHO; 2019. [consultado 17 marzo 2021]. Disponible en: https://apps.who.int/iris/bitstream/handle/10665/325771/WHO-MVP-EMPIAU-2019.06-eng.pdf .

2. Sarukhan A. Ivermectina del suelo a las lombrices y más allá.[Internet] Barcelona: Instituto de Salud Global; 2020. [consultado 17 marzo 2021]. Disponible en https://www.isglobal.org/healthisglobal///custom-blogportlet/ivermectina-del-suelo-a-las-lombrices-y-mas-alla/3098670/0 .

3. Caly L, Druce JD, Catton MG, Jans DA, Wagstaff KM. The FDAapproved drug ivermectin inhibits the replication of SARS-CoV-2 in vitro. Antiviral Res. [Internet]. 2020[consultado 17 marzo 2021];178:104787. Disponible en: https://pubmed.ncbi.nlm.nih.gov/32251768/ Doi: 10.1016/j. antiviral.2020.104787

4. Diawara L, Traoré MO, Badji A, Bissan Y, Doumbia K, Goita SF, et al.Feasibility of onchocerciasis elimination with ivermectin treatment in endemic foci in Africa: first evidence from studies in Mali and Senegal. PLoS Negl Trop Dis. [Internet]. 2009[consultado 17 marzo 2021]; 3(7):e497. Disponible en: https://pubmed.ncbi.nlm.nih.gov/19621091/ doi: 10.1371/journal.pntd.0000497

5. Arévalo AP, Pagotto R, Pórfdo JL., Daghero H., Segovia M., Y masaki $\mathrm{K}$., et al. Ivermectin reduces in vivo coronavirus infection in a mouse experimental model. Sci Rep [Internet]. 2021[consultado 17 marzo 2021];11:7132. Disponible en: https://doi.org/10.1038/s41598-021-866790

6. Carvallo H, Hirsch R, Psaltis A, Contreras V. Study of the efficacy and safety of topical ivermectin + iota- carrageenan in the prophylaxis against COVID-19 in health personnel. J Biomed Res Clin Invest. .Internet] 2020 [consultado 17 marzo 2021];2(1):1007. Disponible en: https://www.
researchgate.net/publication/346034534_Study_of_the_Efficacy_and_ Safety_of_Topical_Ivermectin_lota-_Carrageenan_in_the_Prophylaxis_ against_COVID-19_in_Health_Personnel DOI:10.31546/2633-8653.1007

7. Rajter JC, Sherman M, Naaz F, Vogel F, Sacks J, Rajter JJ. Use of ivermectin is associated withlower mortality in hospitalized patients with coronavirus disease 2019the ivermectin in covid nineteen study. Chest .[Internet] 2021[consultado 17 marzo 2021];159(1):85-92. Disponible en: https://doi org/10.1016/j.chest 202010009

8. Kory P, Medure G, Iglesias J, Varon J, Berkowitz K, Kornfeld H, et al. Review of the emerging evidence demonstrating the efficacy of Ivermectin in the prophylaxis and treatment of COVID-19. OSF [Preprints], [consultado 13 Noviembre 2020]. 2021. [consultado 17 marzo 2021]. Disponible en: https://c19ivermectin.com/kory.html

9. Hill A, Abdulamir A, Ahmed S, Asghar A, Babalola OE, Basri R, et al. Metaanalysis 18 randomized trials of ivermectin to treat SARS-CoV-2 infection. Research Square (Version 1) [Preprint] 2021 [consultado 17 marzo 2021]. Disponible en: https://doi.org/10.21203/rs.3.rs-148845/v1

10. Aguirre Chang G, Trujillo Figueredo A. COVID-19 agudo: tratamiento con dosis altas de ivermectina en casos moderados, severos y críticos. tabla: dosis de ivermectina según severidad, carga viral y respuesta al tratamiento. [consultado 17 marzo 2021]. Disponible en: researchgate.net/ publication/344446953

11. Valerio F, Díaz O, Medina R, Flores J, Paz D, Pineda E, et al. Impact of a multi-mechanism Approach and COVID-19 case fatality rate: the experience of a developing country. ESCMID Conference on Coronavirus Disease. Basel, Suiza. Abstract number 00791. [Internet] 2020 [consultado 17 marzo 2021]. Disponible en: https://www.escmid.org/escmid publication/ escmid elibraryl 